\title{
AN EXACT RESULT FOR HYPERGRAPHS AND UPPER BOUNDS FOR THE TURÁN DENSITY OF $K_{r+1}^{r}{ }^{*}$
}

\author{
LINYUAN $\mathrm{LU}^{\dagger}$ AND YI ZHAO
}

\begin{abstract}
We first answer a question of de Caen [Extremal Problems for Finite Sets, János Bolyai Math. Soc., Budapest, 1994, pp. 187-197]: given $r \geq 3$, if $G$ is an $r$-uniform hypergraph on $n$ vertices such that every $r+1$ vertices span 1 or $r+1$ edges, then $G=K_{n}^{r}$ or $K_{n-1}^{r}$, assuming that $n>(p-1) r$, where $p$ is the smallest prime factor of $r-1$. We then show that the Turán density $\pi\left(K_{r+1}^{r}\right) \leq 1-1 / r-\left(1-1 / r^{p-1}\right)(r-1)^{2} /\left(2 r^{p}\left(\left(\begin{array}{c}r+p \\ p-1\end{array}\right)+\left(\begin{array}{c}r+1 \\ 2\end{array}\right)\right)\right)$, for all even $r \geq 4$, improving a well-known bound $1-\frac{1}{r}$ of de Caen [Ars Combin., 16 (1983), pp. 5-10] and Sidorenko [Vestnik Moskov. Univ. Ser. I Mat. Mekh., 76 (1982), pp. 3-6].
\end{abstract}

Key words. Turán density, extremal problem, hypergraph

AMS subject classifications. 05D05, 05C65

DOI. $10.1137 / 070710615$

1. Introduction. Given a positive integer $n$ and an $r$-uniform hypergraph $H$ (or $r$-graph, for short), the Turán number $\operatorname{ex}(n, H)$ is the maximum number of edges in an $r$-graph on $n$ vertices that does not contain $H$ as a subgraph (called $H$-free). It is easy to see that $f(n, H)=\operatorname{ex}(n, H) /\left(\begin{array}{l}n \\ r\end{array}\right)$ is a decreasing function of $n$ [10]. The limit $\pi(H)=\lim _{n \rightarrow \infty} f(n, H)$, which always exists, is called the Turán density of $H$. Let $K_{k}^{r}$ denote the complete $r$-graph on $k$ vertices. Turán determined ex $\left(n, K_{k}^{2}\right)$ which implies that $\pi\left(K_{k}^{2}\right)=1-\frac{1}{k-1}$ for all $k \geq 3$. However, no Turán density $\pi\left(K_{k}^{r}\right)$ is known for any $k>r \geq 3$. The most well-known case, $k=4$ and $r=3$, is a conjecture of Turán [19], claiming that $\pi\left(K_{4}^{3}\right)=5 / 9$. Erdős [5] offered prizes of $\$ 500$ for determining any $\pi\left(K_{k}^{r}\right)$ with $k>r \geq 3$ and $\$ 1000$ for answering it for all $k$ and $r$. The best (general) known upper bound is due to de Caen [1],

$$
\pi\left(K_{k}^{r}\right) \leq 1-\frac{1}{\left(\begin{array}{c}
k-1 \\
r-1
\end{array}\right)}
$$

and the special case

$$
\pi\left(K_{r+1}^{r}\right) \leq 1-\frac{1}{r}
$$

was also given by Sidorenko [14]. For the lower bound, Sidorenko [17] showed that $\pi\left(K_{r+1}^{r}\right) \geq 1-\frac{\ln r}{2 r}(1+o(1))$ for large $r$. See survey papers of de Caen [2] and Sidorenko [16] for other bounds.

For odd $r \geq 3$, Chung and Lu [4] improved (2) to

$$
\pi\left(K_{r+1}^{r}\right) \leq 1-\frac{5 r+12-\sqrt{9 r^{2}+24 r}}{2 r(r+3)}=1-\frac{1}{r}-\frac{1}{r(r+3)}+O\left(\frac{1}{r^{3}}\right) .
$$

When $r=3$, this gives the best known upper bound $\frac{3+\sqrt{17}}{12} \approx 0.5936$ for $\pi\left(K_{4}^{3}\right)$.

\footnotetext{
* Received by the editors December 11, 2007; accepted for publication (in revised form) June 4, 2009; published electronically August 26, 2009.

http://www.siam.org/journals/sidma/23-3/71061.html

${ }^{\dagger}$ Department of Mathematics, University of South Carolina, Columbia, SC 29208 (lu@math.sc. edu). The research of this author was supported in part by NSF grant DMS-0701111.

${ }^{\ddagger}$ Department of Mathematics and Statistics, Georgia State University, Atlanta, GA 30303 (yzhao6@gsu.edu). The research of this author was supported in part by NSA grants H9823006-1-0140 and H98230-07-1-0019. 
However, it seems more difficult to improve (2) for even $r \geq 4$. For odd $r$, the edge density $f\left(n, K_{r+1}^{r}\right)$ of extremal hypergraphs decreases quickly for small $n$; for example, $f\left(r+2, K_{r+1}^{r}\right)<f\left(r+1, K_{r+1}^{r}\right)$. It is no longer the case for even $r \geq 4$. It is easy to see that

$$
f\left(8, K_{5}^{4}\right)=f\left(7, K_{5}^{4}\right)=f\left(6, K_{5}^{4}\right)=f\left(5, K_{5}^{4}\right)=\frac{4}{5} .
$$

Let $G_{8}^{4}$ be the 4-graph on $[8]=\{1,2, \ldots, 8\}$ with nonedges

$$
\begin{gathered}
\{1,2,3,4\},\{1,2,5,6\},\{1,2,7,8\},\{1,3,5,7\},\{1,3,6,8\},\{1,4,5,8\},\{1,4,6,7\}, \\
\{2,3,5,8\},\{2,3,6,7\},\{2,4,5,7\},\{2,4,6,8\},\{3,4,5,6\},\{3,4,7,8\},\{5,6,7,8\} .
\end{gathered}
$$

It is easy to check that $G_{8}^{4}$ is $K_{5}^{4}$-free with $\frac{4}{5}\left(\begin{array}{l}8 \\ 4\end{array}\right)$ edges, and every 5 vertices on [8] contain exactly 4 edges and one nonedge.

While studying Turán numbers, De Caen [2] asked the following question ${ }^{1}: D e-$ scribe all $r$-graphs such that every $r+1$ vertices span 1 or $r+1$ edges. Equivalently, we consider an $r$-graph $G$, which is the complement of the $r$-graph in de Caen's question:

$$
\text { every } r+1 \text { vertices contain } 0 \text { or } r \text { edges of } G \text {. }
$$

When $r=2$, it is easy to see that $G$ must be a complete bipartite graph (with arbitrary partition sizes). In fact, to prove that $G$ is bipartite, one may consider a shortest odd cycle or apply Lemma 4 in section 3 .

We now answer the question of de Caen for $r \geq 3$. Fix an $n$-vertex set $V$. The empty $r$-graph is the one with no edge; a complete star is an $r$-graph whose edge set consists of all $r$-sets containing some fixed vertex. Clearly the empty graph and complete stars satisfy (4). Theorem 1 below shows that they are the only $r$-graphs satisfying (4) when $|V|$ is not very small. Accordingly their complements, $K_{n}^{r}$ and $K_{n-1}^{r}$, are only $r$-graphs satisfying de Caen's condition.

TheOREM 1. Let $r \geq 3$ and $p$ be the smallest prime factor of $r-1$. Suppose that $G$ is an r-graph on $n$ vertices satisfying (4). If $n>r(p-1)$, then $G$ is either the empty graph or a complete star.

Our proof uses the upper bound for $\operatorname{ex}\left(n, K_{r+1}^{r}\right)$ corresponding to (2). Our construction $G_{8}^{4}$ suggests that a lower bound for $n$ is necessary. A similar problem was solved by Frankl and Füredi [7], who described all 3-graphs such that every 4 vertices contain 0 or 2 edges (the general case when every $r+1$ vertices span 0 or 2 edges is still open).

Let $K_{4}^{3-}$ denote the unique 3 -graph with 4 vertices and 3 edges. It is not hard to prove that $\operatorname{ex}\left(n, K_{4}^{3-}\right) \leq \frac{1}{3}$ (e.g., [1]). Mubayi [13] used the result of Frankl and Füredi [7] and supersaturation to obtain that $\operatorname{ex}\left(n, K_{4}^{3-}\right)<\frac{1}{3}-10^{-6}$. Talbot [18] recently improved the bound to $\frac{1}{3}-\frac{1}{280}$ by considering related chromatic Turán problems. Motivated by these works, we apply Lemma 4, the key step in the proof of Theorem 1, to slightly improve (2) for all $r \geq 3$. (We can also improve (2) by Theorem 1 and supersaturation though the result turns out to be weaker than Theorem 2. See the last section for details.)

TheOREM 2. For $r \geq 3$, let $p$ be the smallest prime factor of $r-1$.

$$
\pi\left(K_{r+1}^{r}\right) \leq \begin{cases}1-\frac{1}{r}-\frac{8}{9 r^{2}}+O\left(\frac{1}{r^{3}}\right), & p=2, \\
1-\frac{1}{r}-\left(1-\frac{1}{r^{p-1}}\right) \frac{(r-1)^{2}}{\left.2 r^{p}\left(\begin{array}{c}
r+p \\
p-1
\end{array}\right)+\left(\begin{array}{c}
r+1 \\
2
\end{array}\right)\right)}, & p \geq 3 .\end{cases}
$$

\footnotetext{
${ }^{1}$ He noted that the $r=2,3$ cases are easy.
} 
When $r$ is odd (thus $p=2$ ), Theorem 2 is slightly weaker than (3). For even $r$, Theorem 2 gives a new upper bound for $\pi\left(K_{r+1}^{r}\right)$. For example, when $r=6 k+4$ (thus $p=3$ ), it gives

$$
\pi\left(K_{r+1}^{r}\right) \leq 1-\frac{1}{r}-\frac{1}{2 r^{3}}+O\left(\frac{1}{r^{4}}\right) .
$$

The rest of the paper is organized as follows. After recalling the proof of (2) in section 2, we prove Theorem 1 in section 3 and Theorem 2 in section 4 . In the last section we give some concluding remarks.

2. Preliminaries. In this section we recall the proof of $(2), \pi\left(K_{r+1}^{r}\right) \leq 1-\frac{1}{r}$, which indicates the source of our improvement. Let $G$ be a $K_{r+1}^{r}$-free $r$-graph. For $0 \leq i \leq r$, let $\Delta_{i}$ denote the family of $(r+1)$-sets that contain exactly $i$ edges of $G$ and $\delta_{i}=\left|\Delta_{i}\right|$.

Lemma 3. For all $n \geq r$,

$$
e x\left(n, K_{r+1}^{r}\right) \leq\left(1-\frac{1}{r}+\frac{1}{r(n-r+1)}\right)\left(\begin{array}{l}
n \\
r
\end{array}\right) .
$$

Moreover, if every $K_{r+1}^{r}$-free $r$-graph $G$ on $n$ vertices satisfies $\sum_{i=1}^{r-1} \delta_{i} \geq y\left(\begin{array}{c}n \\ r+1\end{array}\right)$, then $x=\pi\left(K_{r+1}^{r}\right)$ satisfies

$$
x^{2}-x\left(1-\frac{1}{r}\right)+\frac{(r-1) y}{r(r+1)} \leq 0 .
$$

Proof. Let $G$ be a $K_{r+1}^{r}$-free $r$-graph $G$ on $n$ vertices and $e$ edges. For an $(r-1)$-set $S \subset V(G)$, let $d_{S}=|\{v: S \cup\{v\} \in E(G)\}|$ be the degree of $S$. We have

$$
\begin{aligned}
\sum_{S} d_{S} & =e r, \\
\sum_{S}\left(\begin{array}{c}
d_{S} \\
2
\end{array}\right) & =\sum_{i=1}^{r}\left(\begin{array}{l}
i \\
2
\end{array}\right) \delta_{i}, \\
\sum_{i=1}^{r} i \delta_{i} & =e(n-r) .
\end{aligned}
$$

We derive that

$$
\begin{aligned}
\sum_{S} d_{S}^{2} & =2 \sum_{S}\left(\begin{array}{c}
d_{S} \\
2
\end{array}\right)+\sum_{S} d_{S} \\
& =2 \sum_{i=1}^{r}\left(\begin{array}{l}
i \\
2
\end{array}\right) \delta_{i}+e r \quad \text { using }(7) \text { and }(8) \\
& =\sum_{i} i^{2} \delta_{i}-\sum_{i} i \delta_{i}+e r \\
& =\sum_{i} i^{2} \delta_{i}-e(n-2 r) \text { using }(9) \\
& \leq r \sum_{i=1}^{r} i \delta_{i}-e(n-2 r) \\
& =r e(n-r)-e(n-2 r) .
\end{aligned}
$$

Copyright $@$ by SIAM. Unauthorized reproduction of this article is prohibited. 
Using the Cauchy-Schwarz inequality

$$
\frac{(e r)^{2}}{\left(\begin{array}{c}
n \\
r-1
\end{array}\right)}=\frac{\left(\sum_{S} d_{S}\right)^{2}}{\left(\begin{array}{c}
n \\
r-1
\end{array}\right)} \leq \sum_{S} d_{S}^{2}
$$

and (7), we conclude that

$$
\begin{aligned}
& \frac{(e r)^{2}}{\left(\begin{array}{c}
n \\
r-1
\end{array}\right)} \leq r e(n-r)-e(n-2 r) \\
& \frac{e}{\left(\begin{array}{l}
n \\
r
\end{array}\right)} \leq\left(1-\frac{1}{r}\right)+\frac{1}{r(n-r+1)}
\end{aligned}
$$

with equality holds if all $d_{S}$ are the same and $\sum_{i=1}^{r-1} \delta_{i}=0$. This proves (5).

When $\sum_{i=1}^{r-1} \delta_{i} \geq y\left(\begin{array}{c}n \\ r+1\end{array}\right)$, we can refine the estimate of $\sum d_{S}^{2}$ as

$$
\begin{aligned}
\sum_{S} d_{S}^{2} & =\sum_{i=1}^{r} i^{2} \delta_{i}-e(n-2 r) \\
& =r \sum_{i=1}^{r} i \delta_{i}-\sum_{i=1}^{r-1} i(r-i) \delta_{i}-e(n-2 r) \\
& \leq r \sum_{i=1}^{r} i \delta_{i}-(r-1) \sum_{i=1}^{r-1} \delta_{i}-e(n-2 r) \\
& \leq r e(n-r)-(r-1) y\left(\begin{array}{c}
n \\
r+1
\end{array}\right)-e(n-2 r) .
\end{aligned}
$$

Consequently $\frac{(e r)^{2}}{\left(\begin{array}{c}n \\ r-1\end{array}\right)} \leq r e(n-r)-(r-1) y\left(\begin{array}{c}n \\ r+1\end{array}\right)-e(n-2 r)$, and (6) follows by letting $e=x\left(\begin{array}{l}n \\ r\end{array}\right)$.

3. Proof of Theorem 1. In this section we prove Theorem 1. We need some notations. Let $G=(V, E)$ be an $r$-graph and $T$ be an $(r-1)$-subset of $V$. The neighborhood $N_{T}$ is the set of $x \in V$ such that $\{x\} \cup T \in E$; and let $\bar{N}_{T}=V-T-N_{T}$. We define the degree $d_{T}=\left|N_{T}\right|$ and the nondegree $\bar{d}_{T}=\left|\bar{N}_{T}\right|$. The following lemma provides information on the structures of all $r$-graphs satisfying (4) for $r \geq 2$.

Lemma 4. Let $G$ be an $r$-graph with $r \geq 2$ such that every $r+1$ vertices contain 0 or $r$ edges. The following are true for every $(r-1)$-vertex set $T$.

1. $T \cup \bar{N}_{T}$ contains no edge of $G$.

2. For every vertex $x \in N_{T}$ and every $(r-1)$-subset $D \subseteq T \cup \bar{N}_{T},\{x\} \cup D$ is an edge of $G$.

3. If $r \geq 3$, then either $d_{T}<2$ or $\bar{d}_{T}<p-1$.

Proof. To prove Part 1 , we need to show that for $i=1, \ldots, r$, every $i$-subset of $\bar{N}_{T}$ and every $(r-i)$-subset of $T$ together form a nonedge. We prove this claim by induction on $i$. The definition of $\bar{N}_{T}$ justifies the claim for $i=1$. Suppose the claim holds for some $i \geq 1$. Consider $A \subseteq \bar{N}_{T}$ and $B \subseteq T$ with $|A|=i+1$ and $|B|=r-i-1$. Pick an $(r-i)$-set $C$ such that $B \subset C \subseteq T$. We know that the $r+1$ set $A \cup C$ contains either 0 or $r$ edges, and by induction hypothesis, none of the $i+1(\geq 2) i$-subsets of $A$ together with $C$ form an edge. Therefore $A \cup C$ contains no edge; in particular, $A \cup B$ is not an edge.

Copyright (c) by SIAM. Unauthorized reproduction of this article is prohibited. 
To prove Part 2, we need to show that for $i=0, \ldots, r-1$, every $i$-subset of $\bar{N}_{T}$, every $(r-1-i)$-subset of $T$, and $x$ together form an edge. We again apply induction on $i$. The fact that $x \in N_{T}$ justifies the $i=0$ case. Suppose that $A \subseteq \bar{N}_{T}$ and $B \subseteq T$ with $|A|=i+1$ and $|B|=r-i-2$ for some $i \geq 0$. Pick a set $C$ such that $B \subset C \subseteq T$ with $|C|=r-i-1$. We know that the $(r+1)$-set $A \cup C \cup\{x\}$ contains either 0 or $r$ edges. The induction hypothesis says that all the $i$-subsets of $A$ form edges together with $C \cup\{x\}$. On the other hand, we know from Part 1 that $A \cup C$ is not an edge. Therefore $A \cup C$ is the unique nonedge in $A \cup C \cup\{x\}$, which implies that $A \cup B \cup\{x\}$ is an edge.

To prove Part 3, we assume instead that some $(r-1)$-set $T$ satisfies $d_{T} \geq 2$ and $\bar{d}_{T} \geq p-1$. Let $x, y$ be two vertices in $N_{T}$, and $S$ be an $(r+p-2)$-subset of $T \cup \bar{N}_{T}$. By Part 2, every $(r-1)$-subset of $S$ together with $x$ or $y$ form an edge. This forces that every $(r-1)$-subset of $S$ together with $x, y$ contains precisely $r$ edges. Define an auxiliary $(r-2)$-graph $H$ on $S$ in which an $(r-2)$-subset $R$ of $S$ is an edge if and only if $R \cup\{x, y\}$ is a non-edge of $G$ (here we need $r \geq 3$ ). For $n \geq k>t$, we call a $t$-graph on $n$ vertices an $[n, k, t]$-system if every $k$ vertices contain exactly one edge. Hence $H$ is an $[r+p-2, r-1, r-2]$-system. The number of edges of $H$, $\left(\begin{array}{c}r+p-2 \\ r-1\end{array}\right) / p$ is therefore an integer. This implies that $p$ divides $\left(\begin{array}{c}r+p-2 \\ r-1\end{array}\right)=\left(\begin{array}{c}r+p-2 \\ p-1\end{array}\right)$, or $p$ ! divides $(r+p-2) \cdots r$. Recall that $p$ divides $r-1$ or $r \equiv 1 \bmod p$. We thus obtain a contradiction because $(r+p-2) \cdots r \equiv(p-1) ! \neq 0 \bmod p$.

Proof of Theorem 1. Recall that $r \geq 3$. By Lemma 4, Part 3, every $(r-1)$-subset $T \subset V(G)$ satisfies $d_{T} \leq 1$ or $\bar{d}_{T} \leq p-2$. We claim that if $n>(p-1) r$, then there always exists an $(r-1)$-vertex set $T_{0}$ with $d_{T_{0}} \leq 1$. In fact, suppose that $\bar{d}_{T} \leq p-2$ for all $T$. Then the number of nonedges is at most

$$
\frac{\left(\begin{array}{c}
n \\
r-1
\end{array}\right)(p-2)}{r}=\frac{p-2}{n-r+1}\left(\begin{array}{l}
n \\
r
\end{array}\right)<\left(\frac{1}{r}-\frac{1}{r(n-r+1)}\right)\left(\begin{array}{l}
n \\
r
\end{array}\right)
$$

because $n>(p-1) r$. In other words, $G$ contains more than $\left(1-\frac{1}{r}+\frac{1}{r(n-r+1)}\right)\left(\begin{array}{l}n \\ r\end{array}\right)$ edges. By Lemma 3 , there exists an $(r+1)$-set which contains $r+1$ edges, contradicting (4).

Let $U=T_{0} \cup \bar{N}_{T_{0}}$. Then $|U| \geq n-1$. Lemma 4, Part 1 says that $U$ contains no edges of $G$. If $|U|=n, G$ is the empty graph. Otherwise, $|U|=n-1$. Let $\{x\}=V(G)-U$. Lemma 4, Part 2 implies that $G$ is the complete star with center $x$.

4. Proof of Theorem 2. Suppose that $G=(V, E)$ is a $K_{r+1}^{r}$-free $r$-graph. We call an $(r+1)$-set $S \subseteq V$ bad if $S$ contains $i$ edges of $G$ for some $1 \leq i \leq r-1$. Let $\mathcal{B}$ be the family of all bad sets. Let $\mathcal{Q}$ consist of all $(r+p)$-subsets $Q \subseteq V$ such that some $(r-1)$-subset $S$ of $Q$ has exactly two neighbors in $Q$ (i.e., $\left.\left|N_{S} \cap Q\right|=2\right)$. Then in every induced subhypergraph $G[Q], Q \in \mathcal{Q}$, we have $d_{S}=2$ and $\bar{d}_{S}=p-1$. Lemma 4 , Part 3 thus implies that every $Q \in \mathcal{Q}$ contains some $B \in \mathcal{B}$. For an arbitrary $(r+p)$-set $Q \subseteq V$, we define $f(Q)$ as the number of $(r-1)$-sets $S \subset Q$ such that $\left|N_{S} \cap Q\right|=2$, and $w(Q)$ as the number of bad sets in $Q$. Then $w(Q) \geq 1$ for all $Q \in \mathcal{Q}$. Let $R(G)=\max _{Q \in \mathcal{Q}} f(Q) / w(Q)$. The heart of the proof of Theorem 2 is the lemma below.

Lemma 5. For $r \geq 3$, let $p$ be the smallest prime factor of $r-1$ and

$$
R \geq \max \left\{r-1, R(G): G \text { is a } K_{r+1}^{r} \text {-free } r \text {-graph }\right\}
$$


Then the Turán density $x=\pi\left(K_{r+1}^{r}\right)$ satisfies

$$
x+\frac{r-1}{2 R} x(1-x)^{p-1} \leq 1-\frac{1}{r} .
$$

The proof of Lemma 3 used the Cauchy-Schwarz inequality, or the convexity of $x^{2}$. In order to prove Lemma 5 , we instead use the convexity of $f(x)=x^{2}+c x^{2}(1-x)^{p-1}$ with $0<c \leq 1 / 2$. Since $f(x)>x^{2}$, this convexity helps us to get a tighter bound for $\pi\left(K_{r+1}^{r}\right)$.

Proposition 6. Let $c, p$ be constants satisfying $0 \leq c \leq 1 / 2$ and $p \geq 2$. Then the function $f(x)=x^{2}+c x^{2}(1-x)^{p-1}$ is convex on $[0,1]$.

Proof. First let $p=2$. From $f(x)=x^{2}+c x^{2}(1-x)$, we derive that $f^{\prime \prime}(x)=$ $2+2 c-6 c x$. Since $1-3 x \geq-2$ and $0 \leq c \leq 1 / 2$, we have $f^{\prime \prime}(x) \geq 0$ on $[0,1]$.

Now assume that $p \geq 3$. We have

$$
f^{\prime}(x)=2 x+2 c x(1-x)^{p-1}-c(p-1) x^{2}(1-x)^{p-2}
$$

and

$$
\begin{aligned}
f^{\prime \prime}(x) & =2+2 c(1-x)^{p-1}-4 c(p-1) x(1-x)^{p-2}+c(p-1)(p-2) x^{2}(1-x)^{p-3} \\
& \geq 2+2 c(1-x)^{p-1}-4 c(p-1) x(1-x)^{p-2} \\
& =2+2 c g(x)
\end{aligned}
$$

where $g(x)=(1-x)^{p-1}-2(p-1) x(1-x)^{p-2}=(1-x)^{p-2}(1-(2 p-1) x)$. It is not hard to see that $g(x) \geq-2\left(\frac{2 p-4}{2 p-1}\right)^{p-2}>-2$ on $[0,1]$. Thus $f^{\prime \prime}(x)>2+2 c(-2) \geq 0$ and consequently $f(x)$ is convex on $[0,1]$.

Proof of Lemma 5. Let $G=G(n)$ be a $K_{r+1}^{r}$-free $r$-graph on $n$ vertices with $x(n)\left(\begin{array}{l}n \\ r\end{array}\right)=\operatorname{ex}\left(n, K_{r+1}^{r}\right)$ edges. The fact that $w(Q) \geq 1$ for all $Q \in \mathcal{Q}$ and the definition of $R$ give

$$
\begin{aligned}
\sum_{|S|=r-1}\left(\begin{array}{c}
d_{S} \\
2
\end{array}\right)\left(\begin{array}{c}
\bar{d}_{S} \\
p-1
\end{array}\right) & =\sum_{S} \sum_{Q \supset S:|Q|=r+p,\left|N_{S} \cap Q\right|=2} 1=\sum_{S, Q} \sum_{B \in \mathcal{B}: B \subset Q} \frac{1}{w(Q)} \\
& =\sum_{B \in \mathcal{B}} \sum_{Q \in \mathcal{Q}: B \subset Q} \frac{f(Q)}{w(Q)} \leq R|\mathcal{B}|\left(\begin{array}{c}
n-r+1 \\
p-1
\end{array}\right) .
\end{aligned}
$$

We thus obtain a bound for the number of bad sets:

$$
|\mathcal{B}|=y\left(\begin{array}{c}
n \\
r+1
\end{array}\right) \geq \frac{\sum_{S}\left(\begin{array}{c}
d_{S} \\
2
\end{array}\right)\left(\begin{array}{c}
\bar{d}_{S} \\
p-1
\end{array}\right)}{\left(\begin{array}{c}
n-r+1 \\
p-1
\end{array}\right) R} .
$$

We define a function $f(x)=x^{2}+c x^{2}(1-x)^{p-1}$ with $c=\frac{r-1}{2 R}$. The definition of $R$ forces $c \leq \frac{1}{2}$. By Proposition $6, f(x)$ is convex on $[0,1]$. For every $(r-1)$-set $S$, let $x_{S}=\frac{d_{S}}{n-r+1}$. The convexity of $f$ gives

$$
f\left(\frac{\sum_{S} x_{S}}{\left(\begin{array}{c}
n \\
r-1
\end{array}\right)}\right) \leq \frac{\sum_{S} f\left(x_{S}\right)}{\left(\begin{array}{c}
n \\
r-1
\end{array}\right)} .
$$

Since $\frac{\sum_{S} x_{S}}{\left(\begin{array}{c}n \\ r-1\end{array}\right)}=\frac{\sum_{S} d_{S}}{(n-r+1)\left(\begin{array}{c}n \\ r-1\end{array}\right)}=\frac{e}{\left(\begin{array}{l}n \\ r\end{array}\right)}=x(n)$, we have $f\left(\frac{\sum_{S} x_{S}}{\left(\begin{array}{c}n \\ r-1\end{array}\right)}\right)=f(x(n))$.

Copyright (c) by SIAM. Unauthorized reproduction of this article is prohibited. 
On the other hand,

$\frac{\sum_{S} f\left(x_{S}\right)}{\left(\begin{array}{c}n \\ r-1\end{array}\right)}=\frac{\sum_{S}\left(x_{S}^{2}+c x_{S}^{2}\left(1-x_{S}\right)^{p-1}\right)}{\left(\begin{array}{c}n \\ r-1\end{array}\right)}=\frac{\sum_{S} d_{S}^{2}}{(n-r+1)^{2}\left(\begin{array}{c}n \\ r-1\end{array}\right)}+c \frac{\sum_{S} d_{S}^{2} \bar{d}_{S}^{p-1}}{(n-r+1)^{p+1}\left(\begin{array}{c}n \\ r-1\end{array}\right)}$.

Using (10),

$$
\begin{aligned}
\frac{\sum_{S} d_{S}^{2}}{(n-r+1)^{2}\left(\begin{array}{c}
n \\
r-1
\end{array}\right)} & \leq \frac{r e(n-r)-(r-1) y\left(\begin{array}{c}
n \\
r+1
\end{array}\right)-e(n-2 r)}{(n-r+1)^{2}\left(\begin{array}{c}
n \\
r-1
\end{array}\right)} \\
& =\frac{r e(n-r)-e(n-2 r)}{(n-r+1)^{2}\left(\begin{array}{c}
n \\
r-1
\end{array}\right)}-\frac{r-1}{(r+1) r} y+O\left(\frac{1}{n}\right) .
\end{aligned}
$$

Since $d_{S}, \bar{d}_{S} \leq n$ for all $S$, we have $\sum_{S} d_{S}^{2} \bar{d}_{S}^{p-1}=O\left(n^{p+r-1}\right)+\sum_{S} 2(p-1) !\left(\begin{array}{c}d_{S} \\ 2\end{array}\right)\left(\begin{array}{c}\bar{d}_{S} \\ p-1\end{array}\right)$ and

$$
\begin{aligned}
c \frac{\sum_{S} d_{S}^{2} \bar{d}_{S}^{p-1}}{(n-r+1)^{p+1}\left(\begin{array}{c}
n \\
r-1
\end{array}\right)} & =\frac{O\left(n^{p+r-1}\right)+\sum_{S} 2 c(p-1) !\left(\begin{array}{c}
d_{S} \\
2
\end{array}\right)\left(\begin{array}{c}
\bar{d}_{S} \\
p-1
\end{array}\right)}{(n-r+1)^{p+1}\left(\begin{array}{c}
n \\
r-1
\end{array}\right)} \\
& \leq 2 c(p-1) ! \frac{R\left(\begin{array}{c}
n-r+1 \\
p-1
\end{array}\right) y\left(\begin{array}{c}
n \\
r+1
\end{array}\right)}{(n-r+1)^{p+1}\left(\begin{array}{c}
n \\
r-1
\end{array}\right)}+O\left(\frac{1}{n}\right) \\
& =2 c \frac{R}{(r+1) r} y+O\left(\frac{1}{n}\right) \\
& =\frac{r-1}{(r+1) r} y+O\left(\frac{1}{n}\right) .
\end{aligned}
$$

Putting these together, we have

$$
\begin{aligned}
f(x(n)) \leq \frac{\sum_{S} f\left(x_{S}\right)}{\left(\begin{array}{c}
n \\
r-1
\end{array}\right)} & \leq \frac{r e(n-r)-e(n-2 r)}{(n-r+1)^{2}\left(\begin{array}{c}
n \\
r-1
\end{array}\right)}+O\left(\frac{1}{n}\right) \\
& =\left(1-\frac{1}{r}\right) x(n)+O\left(\frac{1}{n}\right) .
\end{aligned}
$$

The claim (12) follows by letting $n \rightarrow \infty$ and substituting $f(x)=x^{2}+\frac{r-1}{2 R} x^{2}$ $(1-x)^{p-1}$. प

Trivially we can choose $R=\left(\begin{array}{l}r+p \\ r-1\end{array}\right)$-applying it in (12) already yields a better bound for $\pi\left(K_{r+1}^{r}\right)$ than (2). The following technical lemma refines our bound for $R$.

Lemma 7. For any $K_{r+1}^{r}$-free $r$-graph $G$ with at least $r+p$ vertices,

$$
R(G) \leq \begin{cases}\left(\frac{3 r+2}{4}\right)^{2}, & p=2 \\
\left(\begin{array}{c}
r+1 \\
2
\end{array}\right)+\left(\begin{array}{c}
r+p \\
p-1
\end{array}\right), & p \geq 3\end{cases}
$$

Proof. Fix $Q \in \mathcal{Q}$. Let $q=r+p=|Q|$. We consider a $p$-graph $H$ on $Q$ whose edges are the complements of edges of $G$, namely,

$$
E(H)=\{Q-e: e \in E(G), e \subset Q\} .
$$

Therefore a $(p-1)$-subset $S \subset Q$ has degree $i$ in $H$ if and only if the $(r+1)$-set $Q \backslash S$ contains exactly $i$ edges of $G$. Let $W$ be the family of $(p-1)$-sets with degree $i$ (in $H$ ) 
for some $1 \leq i \leq r-1$ (their complementary sets in $Q$ are bad sets for $G$ ), and $F$ be the set of $(p+1)$-sets containing exactly two edges of $H$ (their complementary sets in $Q$ have degree 2 in $G[Q]$ ). Following the definition of $w(Q)$ and $f(Q)$ in the beginning of the section, we have $|W|=w(Q)$ and $|F|=f(Q)$. Note that the degree of any $(p-1)$-set in $H$ is at most $r$ since $G$ is $K_{r+1}^{r}$-free. We also know that $|W|=w(Q) \geq 1$ since $Q$ contains at least one bad set. From now on we consider $H$ as our underlying hypergraph (instead of $G$ ). Our goal is to obtain upper bounds for $|F| /|W|$.

The $p=2$ case. Partition the vertex set $Q$ into $W=\left\{x: 1 \leq d_{\{x\}} \leq r-1\right\}$, $U=\left\{x: d_{\{x\}}=r\right\}$, and $Z=\left\{x: d_{\{x\}}=0\right\}$. Since each vertex in $U$ misses at most one vertex of $Q$, nonedges inside $U$ form a matching $M$. Let $U_{1}=U-V(M)$ consist of vertices in $U$ not covered by $M$. The family $F$ consists of all triples $T=a b c$ such that $a b, a c \in E(H)$ and $b c \notin E(H)$. Clearly $T \subset W \cup U$ for all $T \in F$.

We partition $F=F_{0} \cup F_{1} \cup F_{2}$ such that $F_{0}$ consists of all $T \in F$ with $|T \cap W| \geq 2$, $F_{1}$ consists of those $T=a b c$ with $b, c \in U$, and $F_{2}$ consists of those $a b c$ with exactly one of $b, c$ in $W$. We have $\left|F_{0}\right| \leq\left(\begin{array}{c}|W| \\ 2\end{array}\right)(q-2)$, and $\left|F_{1}\right| \leq|M|(q-2)$. Since $|M| \leq$ $\left(q-\left|U_{1}\right|-|W|\right) / 2$ and $|W| \geq 1$,

$$
\begin{aligned}
\left|F_{0}\right|+\left|F_{1}\right| & \leq\left(\begin{array}{c}
|W| \\
2
\end{array}\right)(q-2)+\frac{1}{2}\left(q-\left|U_{1}\right|-|W|\right)(q-2) \\
& \leq \frac{1}{2}|W|(q-2)\left(\frac{1}{2}(|W|-1)+\left(q-\left|U_{1}\right|-|W|\right)\right) \\
& =\frac{1}{2}|W|(q-2)\left(q-\left|U_{1}\right|-1\right) .
\end{aligned}
$$

To estimate $F_{2}$, let us consider a vertex $x \in W$. By definition of $M, x$ is adjacent to all the vertices in $V(M)$. If $x$ has $t$ nonneighbors in $U$, then $t \leq\left|U_{1}\right|$ and the number of triples of $F_{2}$ containing $x$ is at most

$$
t(|U|-t) \leq \begin{cases}\left|U_{1}\right|\left(|U|-\left|U_{1}\right|\right) & \text { if }\left|U_{1}\right| \leq|U| / 2 \\ |U|^{2} / 4 & \text { otherwise }\end{cases}
$$

First assume that $\left|U_{1}\right| \leq|U| / 2$. Since $|U| \leq q-1$, we have

$$
\left|F_{2}\right| \leq|W|\left|U_{1}\right|\left(|U|-\left|U_{1}\right|\right) \leq|W|\left|U_{1}\right|\left(p-1-\left|U_{1}\right|\right) .
$$

Together with (14) and using $|W| \geq 1$, we derive that

$$
\begin{aligned}
|F| & \leq|W|\left(\frac{1}{2}(q-2)\left(q-\left|U_{1}\right|-1\right)+\left|U_{1}\right|\left(q-1-\left|U_{1}\right|\right)\right) \\
& \leq|W|\left(\frac{3 q}{4}-1\right)^{2},
\end{aligned}
$$

where equality holds when $\left|U_{1}\right|=q / 4$. In contrast, when $\left|U_{1}\right|>|U| / 2$, we have

$$
\begin{aligned}
|F| & \leq|W|\left(\frac{1}{2}(q-2)\left(q-\left|U_{1}\right|-1\right)+\frac{1}{4}|U|^{2}\right) \\
& \leq|W|\left(\frac{1}{2}(q-2)\left(q-\frac{1}{2}|U|-1\right)+\frac{1}{4}|U|^{2}\right) \\
& \leq \frac{1}{4}|W|(q-1)(2 q-3),
\end{aligned}
$$

Copyright (c) by SIAM. Unauthorized reproduction of this article is prohibited. 
where equality holds when $|U|=q-1$ and $\left|U_{1}\right|=|U| / 2$. It is easy to check that $\left(\frac{3 q}{4}-1\right)^{2} \geq \frac{1}{4}(q-1)(2 q-3)$ for any $q$. We therefore conclude that $|F| /|W| \leq$ $\left(\frac{3 q}{4}-1\right)^{2}=\left(\frac{3 r+2}{4}\right)^{2}$.

The $p \geq 3$ case. Partition $F$ into $F_{1}=\{S \in F: S \supset T$ for some $T \in W\}$ and $F_{2}=F-F_{1}$. Clearly $\left|F_{1}\right| \leq|W|\left(\begin{array}{c}q-p+1 \\ 2\end{array}\right)$. We prove below that $\left|F_{2}\right| \leq \sqrt{|W|}\left(\begin{array}{c}q \\ p-1\end{array}\right)$.

Fix a $(p+1)$-set $S \in F_{2}$. By the definition of $F_{2}$, all $(p-1)$-subsets of $S$ have either degree 0 or degree $r$ in $H$ (equivalently, nondegree 1), and $S$ contains exactly two edges ( $p$-sets) of $H$. We denote these two edges by $T \cup\{x\}$ and $T \cup\{y\}$, where $T \subset S$ is a $(p-1)$-set. We have two observations.

ObSERVATion 1. For any $a \in T$, the $(p-1)$-set $T \cup\{x\}-\{a\}$ must have degree $r$ since $T \cup\{x\} \in E(H)$ and $S-\{a\} \notin E(H)$. This implies that $T \cup\{x, u\}-\{a\} \in E(H)$ for any vertex $u \notin S$.

ObServation 2. For two distinct vertices $a, b \in T$, the $(p-1)$-set $S-\{a, b\}$ must have degree 0 since two p-sets $S-\{a\}, S-\{b\}$ are not edges. Note that we need $|T| \geq 2$ or $p \geq 3$ here.

We define a function $g: F_{2} \rightarrow\left(\begin{array}{c}Q \\ p-1\end{array}\right)$ such that $g(S)=T$. If $g$ is a one-to-one function, then $\left|F_{2}\right| \leq\left(\begin{array}{c}q \\ p-1\end{array}\right) \leq \sqrt{|W|}\left(\begin{array}{c}q \\ p-1\end{array}\right)$. Otherwise, let $T$ be a $(p-1)$-set with the maximum size of $g^{-1}(T)=\left\{S \in F_{2}: g(S)=T\right\}$. Let $M$ consist of all 2-sets $S-T$ such that $S \in g^{-1}(T)$. Then $|M|=\left|g^{-1}(T)\right| \geq 2$.

We first claim that $M$ is a matching; namely, any two pairs in $M$ are disjoint. Suppose instead $x y, x z \in M$. For any $a \in T$, the $(p-1)$-set $\{x\} \cup T-\{a\}$ is contained in two nonedges $\{x, y\} \cup T-\{a\}$ and $\{x, z\} \cup T-\{a\}$, contradicting Observation 1. We next claim that $\{x, y\} \cup T-\{a, b\} \in W$ for two pairs $P, Q \in M$, any vertices $x \in P, y \in Q$, and any two vertices $a, b \in T$. In fact, Observation 2 says that $T_{P}=P \cup T-\{a, b\}$ has degree 0 , and consequently $\{y\} \cup T_{P}$ is a nonedge. Similarly $\{x\} \cup T_{Q}$ is also a nonedge with $T_{Q}=Q \cup T-\{a, b\}$. On the other hand, we know that $\{x, y\} \cup T-\{a\}$ is an edge from Observation 1. Hence $\{x, y\} \cup T-\{a, b\}$ has degree at least 1 and nondegree at least 2 , and thus it belongs to $W$. This implies that $|W| \geq 2^{2}\left(\begin{array}{c}|M| \\ 2\end{array}\right)\left(\begin{array}{c}p-1 \\ 2\end{array}\right) \geq|M|^{2}$ (since $|M| \geq 2$ ). We thus conclude that $\sum_{T \in R_{2}}\left|g^{-1}(T)\right| \leq \sqrt{|W|}\left(\begin{array}{c}q \\ p-1\end{array}\right)$.

Consequently $|F| \leq|W|\left(\begin{array}{c}q-p+1 \\ 2\end{array}\right)+\sqrt{|W|}\left(\begin{array}{c}q \\ p-1\end{array}\right)$ and $|F| /|W| \leq\left(\begin{array}{c}q-p+1 \\ 2\end{array}\right)+$ $\left(\begin{array}{c}q \\ p-1\end{array}\right)$.

Proof of Theorem 2. Let $R$ be the upper bound for $R(G)$ in Lemma 7:

$$
R= \begin{cases}\left(\frac{3 r+2}{4}\right)^{2}, & p=2, \\
\left(\begin{array}{c}
r+1 \\
2
\end{array}\right)+\left(\begin{array}{c}
r+p \\
p-1
\end{array}\right), & p \geq 3 .\end{cases}
$$

It is easy to check that $R \geq r-1$ and consequently $c=\frac{r-1}{2 R} \leq \frac{1}{2}$. Let the Turán density $x=\pi\left(n, K_{r+1}^{r}\right)=1-\frac{1}{r}-\varepsilon$. The inequality (12) from Lemma 5 gives

$$
\varepsilon \geq c\left(1-\frac{1}{r}-\varepsilon\right)\left(\frac{1}{r}+\varepsilon\right)^{p-1}>c\left(1-\frac{1}{r}-\varepsilon\right)\left(\frac{1}{r}\right)^{p-1}
$$

which implies that

$$
\begin{aligned}
\varepsilon & >\left(1-\frac{1}{r}\right) \frac{c}{c+r^{p-1}} \\
& >\left(1-\frac{1}{r}\right) \frac{c}{r^{p-1}}\left(1-\frac{c}{r^{p-1}}\right) .
\end{aligned}
$$

Copyright (c) by SIAM. Unauthorized reproduction of this article is prohibited. 
We now apply the definition of $R$ to $c=\frac{r-1}{2 R}$. When $p=2$, this gives $\varepsilon>\frac{8}{9 r^{2}}+O\left(1 / r^{3}\right)$. When $p \geq 3$, we have

$$
\begin{gathered}
\varepsilon>\left(1-\frac{1}{r}\right) \frac{r-1}{2 r^{p-1}\left(\left(\begin{array}{c}
r+p \\
p-1
\end{array}\right)+\left(\begin{array}{c}
r+1 \\
2
\end{array}\right)\right)}\left(1-\frac{c}{r^{p-1}}\right) \\
>\frac{(r-1)^{2}}{2 r^{p}\left(\left(\begin{array}{c}
r+p \\
p-1
\end{array}\right)+\left(\begin{array}{c}
r+1 \\
2
\end{array}\right)\right)}\left(1-\frac{1}{r^{p-1}}\right) \cdot
\end{gathered}
$$

\section{Concluding remarks.}

- Following the approach of Mubayi [13], we can improve (2) by Theorem 1 and supersaturation as follows. By Theorem 1, every $K_{r+1}^{r}$-free $r$-graph on $(p-1) r+1$ vertices either contains a bad set or has at most $\left(\begin{array}{c}(p-1) r \\ r-1\end{array}\right)$ edges. In fact, from a simple removing argument and the structure of the $r$-graphs that satisfy (4), we can show that every $K_{r+1}^{r}$-free $r$-graph on $n_{0}=(p-1)(r+1)$ vertices either contains $p-1$ bad sets or has at most $\left(\begin{array}{c}n_{0}-1 \\ r-1\end{array}\right)$ edges. By averaging arguments and (5), every $K_{r+1}^{r}$-free $r$-graph with $n \geq n_{0}$ vertices and $x\left(\begin{array}{l}n \\ r\end{array}\right)$ edges contains at least

$$
\frac{x-\frac{r}{n_{0}}}{1-\frac{1}{r}+\frac{1}{r\left(n_{0}-r+1\right)}-\frac{r}{n_{0}}} \frac{p-1}{\left(\begin{array}{c}
n_{0} \\
r+1
\end{array}\right)}\left(\begin{array}{c}
n \\
r+1
\end{array}\right)
$$

bad sets - this technique is usually called supersaturation [6]. By (6) in Lemma 3 , as $r \rightarrow \infty$,

$$
\pi\left(K_{r+1}^{r}\right) \leq 1-\frac{1}{r}-(1+o(1)) \frac{p-2}{\left(\begin{array}{c}
(p-1)(r+1) \\
r+1
\end{array}\right) r(p-1)},
$$

which is weaker than Theorem 2. This suggests that (13) gives a better estimate on the number of bad sets than the one from supersaturation.

- The bound $n>r(p-1)$ in Theorem 1 is tight for odd $r$ and $r=4$, but we do not know if it is tight for any even number $r>4$. The bound for $R$ in Lemma 7 is near tight for $p=2$ and tight up to a constant factor for $p=3$, but we do not know if it is tight for $p>3$.

- Let us consider $K_{5}^{4}$. The following bounds are due to Giraud [8, 9].

$$
\frac{11}{16} \leq \pi\left(K_{5}^{4}\right) \leq \frac{3}{4}
$$

Sidorenko showed [14] that $\pi\left(K_{5}^{4}\right) \leq 0.74912$ and conjectured [16] that the lower bound above gives the correct value. Applying (12) with $R=13$ (the proof of $R=13$ is not very hard but not simple either), we derive that $\pi\left(K_{5}^{4}\right) \leq 0.74439$. Markström [12] recently obtained a much better bound $\pi\left(K_{5}^{4}\right) \leq 0.73655$ by determining $\operatorname{ex}\left(n, K_{5}^{4}\right)$ for $n \leq 16$ and applying the simple bound $\pi\left(K_{5}^{4}\right) \leq \operatorname{ex}\left(n, K_{5}^{4}\right) /\left(\begin{array}{l}n \\ 4\end{array}\right)\left(\operatorname{ex}\left(n, K_{5}^{4}\right)\right.$ was only known [3] for $n \leq$ 10 before).

- For any $r$-graph $F$ with $f$ edges, Sidorenko [15] used an analytic approach to obtain

$$
\pi(F) \leq 1-\frac{1}{f-1}
$$

Copyright ( $\odot$ by SIAM. Unauthorized reproduction of this article is prohibited. 
When $F=K_{r+1}^{r}, f=r+1$, and this gives (2). An $r$-graph is called a forest if there is an ordering of its edges $E_{1}, \ldots, E_{t}$ such that for every $i=2, \ldots, t$, there exists $j_{i}<i$ such that $\left(E_{i} \cap \cup_{\ell=1}^{i-1} E_{\ell}\right) \subseteq E_{j_{i}}$. Keevash [11] found that the method of Sidorenko actually implies that $\pi=\pi(F)$ satisfies

$$
\pi^{t-1}+(f-t)(\pi-1) \leq 0,
$$

where $t$ is the size of a largest forest contained in $F$. When $t=2,(17)$ reduces to (16); when $t>2$, (17) improves (16). In our case (17) does not improve the $1-1 / r$ bound for $\pi\left(K_{r+1}^{r}\right)$ because the largest forest in $K_{r+1}^{r}$ has only two edges.

Acknowledgments. The authors thank the referees for their comments that improved the presentation.

\section{REFERENCES}

[1] D. DE CAEn, Extension of a theorem of Moon and Moser on complete subgraphs, Ars Combin., 16 (1983), pp. $5-10$.

[2] D. DE CAEn, The current status of Turán's problem on hypergraphs, in Extremal Problems for Finite Sets (Visegrád, 1991), Bolyai Soc. Math. Stud., 3, János Bolyai Math. Soc., Budapest, 1994, pp. 187-197.

[3] D. de Caen, D. L. Kreher, S. P. Radziszowski, and W. H. Mills, On the coverings t-sets with $t+1$-sets: $C(9,5,4)$ and $C(10,6,5)$, Discrete Math., 92 (1991), pp. 65-77.

[4] F. Chung And L. Lu, An upper bound for the Turán number $t_{3}(n, 4)$, J. Combin. Theory Ser. A, 87 (1999), pp. 381-389.

[5] P. ERDős, On the combinatorial problems which I would like to see solved, Combinatorica, 1 (1981), pp. 25-42.

[6] P. ERdös AND M. Simonovits, Supersaturated graphs and hypergraphs, Combinatorica, 3 (1983), pp. 181-192.

[7] P. Frankl and Z. Füredi, An exact result for 3-graphs, Discrete Math., 50 (1984), pp. 323328.

[8] G. Giraud, Majoration du nombre de Ramsey ternaire-bicolore en (4, 4), C. R. Acad. Sci. Paris Sér. A-B, 269 (1969), pp. A620-A622.

[9] G. GiRaud, Remarques sur deux problèmes extrémaux, Discrete Math., 84 (1990), pp. 319-321.

[10] G. Katona, T. Nemetz, And M. Simonovits, On a problem of Turán in the theory of graphs, Mat. Lapok, 15 (1964), pp. 228-238.

[11] P. Keevash, The Turán problem for hypergraphs on fixed size, Electron. J. Combin., 12 (2005), Note 11, pp. 1-6.

[12] K. Markström, Extremal graphs and bounds for the Turan density of the 4-uniform $\mathbf{K}_{5}$, Discrete Math., 309 (2009), pp. 5231-5234.

[13] D. MuBAyI, On hypergraphs with every four points spanning at most two triples, Electron. J. Combin., 10 (2003), Note 10, pp. 1-4.

[14] A. Sidorenko, The method of quadratic forms in a combinatorial problem of Turán, Vestnik Moskov. Univ. Ser. I Mat. Mekh., 76 (1982), pp. 3-6 (in Russian).

[15] A. Sidorenko, An analytic approach to extremal problems for graphs and hypergraphs, in Extremal Problems for Finite Sets (Visegrád, 1991), Bolyai Soc. Math. Stud., 3, János Bolyai Math. Soc., Budapest, 1994, pp. 423-455.

[16] A. Sidorenko, What we know and what we do not know about Turán numbers, Graphs Combin., 11 (1995), pp. 179-199.

[17] A. Sidorenko, Upper bounds for Turán numbers, J. Combin. Theory Ser. A, 77 (1997), pp. 134147.

[18] J. TALBOT, Chromatic Turán problems and a new upper bound for the Turán density of $\mathcal{K}_{4}^{-}$, European J. Combin., 28 (2007), pp. 2125-2142.

[19] P. Turán, On an extremal problem in graph theory, Mat. Fiz. Lapok, 48 (1941), pp. 436-452. 